\title{
HPM and ADM for partial differential equations
}

\author{
Jafar. Biazar*, Fereshteh. Goldoust \\ Department of Applied Mathematics, Faculty of Mathematical Sciences, University of Guilan, P.O. Box 41335- 1914, Rasht, Iran \\ *Corresponding author E-mail: biazar@guilan.ac.ir
}

\begin{abstract}
In this work, Homotopy Perturbation method and Adomian decomposition method are used to solve systems of partial differential equations. These methods are which are well known methods nowadays based upon perturbation and decomposition theories. Some examples are provided to show the ability of these methods.
\end{abstract}

Keywords: Homotopy perturbation method; Adomian decomposition method; Systems of partial differential equations.

\section{Introduction}

The Homotopy perturbation method introduced by He [1], in 1998. Recently a great deal of interest has been focused on the method and it's applications. Method, well addressed in [1, 2]. In this method the solution is considered as the summation of an infinite series, which usually converges rapidly to the exact solution. To illustrate the basic concepts of this method, consider the following nonlinear differential equations.

$A(u)-f(r)=0, \quad r \in \Omega$.

With boundary conditions

$B\left(u, \frac{\partial u}{\partial n}\right)=0, \quad r \in \Gamma$.

Where $\mathrm{A}$ is a general differential operator, $B$ is a boundary operator, $f(r)$ is a known analytic operator, and $\Gamma$ is the boundary of the domain $\Omega$.

Generally speaking the operator A can be divided into two parts L, and N, where L is linear, and $\mathrm{N}$ is a nonlinear operator Eq.(1), therefore, can be rewritten as follows:

$L(u)+N(u)-f(r)=0$.

Let's construct the homotopy $V(r, p): \Omega \times[0,1] \rightarrow \mathfrak{R}$, which satisfies:

$H(v, p)=(1-p)\left[L(v)-L\left(u_{0}\right)\right]+p[A(v)-f(r)]=0, r \in \Omega$.

Where $u_{0}$ is an initial approximation of the solution of Eq. (1).

And in continue we provided the basic idea about Adomian decomposition method. The Adomian decomposition method is a technique for solving functional equation in the following canonical form [3, 4]:

$u=f+G(u)$.

The solution $u$ is considered as the summation of a series, say:

$u=\sum_{n=0}^{\infty} u_{n}$.

And $\mathrm{G}(\mathrm{u})$ as the summation of the following series,

$G(u)=\sum_{n=0}^{\infty} A_{n}\left(u_{0}, . ., u_{n}\right)$.

Where $A_{n}$ is called Adomian polynomials, has been introduced by Adomian, as the following:

$A_{n}\left(u_{0}, . ., u_{n}\right)=\left.\frac{1}{n !} \frac{d^{n}}{d p^{n}}\left[G\left(\sum_{i=0}^{n} u_{i} p^{i}\right)\right]\right|_{p=0}$.

And for functional equations, with several variables, the following extension of (8) can be used. 
$A_{n}\left(u_{10}, . ., u_{1 n}, u_{20}, . ., u_{2 n}, . ., u_{m 0}, . ., u_{m n}\right)=\left.\frac{1}{n !} \frac{d^{n}}{d p^{n}}\left[G\left(\sum_{n=0}^{\infty} u_{1 n} p_{n}, . ., \sum_{n=0}^{\infty} u_{m n} p_{n}\right)\right]\right|_{p=0}$.

Where $G\left(u_{1}, . ., u_{n}\right)$ is a functional depending on $\mathrm{n}$ variables, each of them is an unknown function which are considered as the summation of series say,

$u_{j}=\sum_{n=0}^{\infty} u_{j n} p_{n}, \quad j=0,1, \ldots, n$

\section{Solution of a system of PDE by HPM and ADM.}

Consider the following system of partial differential equations:

$$
\begin{aligned}
& \frac{\partial u_{1}}{\partial t}+\frac{\partial u_{2}}{\partial x_{1}}+\ldots+\frac{\partial u_{n}}{\partial x_{n-1}}=g_{1}, \\
& \frac{\partial u_{2}}{\partial t}+\frac{\partial u_{1}}{\partial x_{1}}+\ldots+\frac{\partial u_{n}}{\partial x_{n-1}}=g_{2} \\
& \vdots \\
& \frac{\partial u_{n}}{\partial t}+\frac{\partial u_{2}}{\partial x_{1}}+\ldots+\frac{\partial u_{1}}{\partial x_{n-1}}=g_{n}
\end{aligned}
$$

With initial condition:

$$
\begin{aligned}
& u_{1}\left(x_{1}, x_{2}, . ., x_{n-1}, 0\right)=f_{1}\left(x_{1}, x_{2}, . ., x_{n-1}\right), \\
& u_{2}\left(x_{1}, x_{2}, . ., x_{n-1}, 0\right)=f_{2}\left(x_{1}, x_{2}, . ., x_{n-1}\right), \\
& \vdots \\
& u_{n}\left(x_{1}, x_{2}, . ., x_{n-1}, 0\right)=f_{n}\left(x_{1}, x_{2}, . ., x_{n-1}\right) .
\end{aligned}
$$

Where $g_{1}, g_{2}, . ., g_{n}$ are inhomogeneous terms. To solve system (10), by Homotopy perturbation method, let' s constructs the following homotopies [5],

$$
\begin{aligned}
& (1-p)\left(\frac{\partial U_{1}}{\partial t}-\frac{\partial u_{10}}{\partial t}\right)+p\left(\frac{\partial U_{1}}{\partial t}+\frac{\partial U_{2}}{\partial x_{1}}+\ldots+\frac{\partial U_{n}}{\partial x_{n-1}}-g_{1}\right)=0 \\
& (1-p)\left(\frac{\partial U_{2}}{\partial t}-\frac{\partial u_{20}}{\partial t}\right)+p\left(\frac{\partial U_{2}}{\partial t}+\frac{\partial U_{1}}{\partial x_{1}}+\ldots+\frac{\partial U_{n}}{\partial x_{n-1}}-g_{2}\right)=0 \\
& \vdots \\
& (1-p)\left(\frac{\partial U_{n}}{\partial t}-\frac{\partial u_{n 0}}{\partial t}\right)+p\left(\frac{\partial U_{n}}{\partial t}+\frac{\partial U_{2}}{\partial x_{1}}+\ldots+\frac{\partial U_{1}}{\partial x_{n-1}}-g_{n}\right)=0
\end{aligned}
$$

Let's present the solution of the system (6), $\frac{\partial U_{1}}{\partial t}=\frac{\partial U_{10}}{\partial t}+p \frac{\partial U_{11}}{\partial t}+p^{2} \frac{\partial U_{12}}{\partial t}+\ldots$ as the following:

$$
\begin{aligned}
& U_{1}=U_{10}+p U_{11}+p^{2} U_{12}+\ldots \\
& U_{2}=U_{20}+p U_{21}+p^{2} U_{22}+\ldots \\
& \vdots \\
& U_{n}=U_{n 0}+p U_{n 1}+p^{2} U_{n 2}+\ldots
\end{aligned}
$$

Substituting (12) in to (11), and equating the coefficients of the terms with identical powers of $\mathrm{p}$, leads to: 


$$
\begin{aligned}
& p^{0}:\left\{\begin{array}{l}
\frac{\partial U_{10}}{\partial t}-\frac{\partial u_{10}}{\partial t}=0 \\
\frac{\partial U_{20}}{\partial t}-\frac{\partial u_{20}}{\partial t}=0 \\
\vdots \\
\frac{\partial U_{n 0}}{\partial t}-\frac{\partial u_{n 0}}{\partial t}=0
\end{array}\right. \\
& p^{1}:\left\{\begin{array}{l}
\frac{\partial U_{11}}{\partial t}+\frac{\partial u_{10}}{\partial t}+\frac{\partial U_{20}}{\partial x_{1}}+\ldots \frac{\partial U_{n 0}}{\partial x_{n-1}}-g_{1}=0, \\
\frac{\partial U_{22}}{\partial t}+\frac{\partial u_{20}}{\partial t}+\frac{\partial U_{10}}{\partial x_{1}}+\ldots \frac{\partial U_{n 0}}{\partial x_{n-1}}-g_{2}=0, \\
\vdots \\
\frac{\partial U_{n 1}}{\partial t}+\frac{\partial u_{10}}{\partial t}+\frac{\partial U_{20}}{\partial x_{1}}+\ldots \frac{\partial U_{10}}{\partial x_{n-1}}-g_{n}=0 .
\end{array}\right. \\
& p^{2}:\left\{\begin{array}{l}
\frac{\partial U_{12}}{\partial t}+\frac{\partial U_{21}}{\partial x_{1}}+\ldots \frac{\partial U_{n 1}}{\partial x_{n-1}}=0 \\
\frac{\partial U_{22}}{\partial t}+\frac{\partial U_{11}}{\partial x_{1}}+\ldots \frac{\partial U_{n 1}}{\partial x_{n-1}}=0 \\
\vdots \\
\frac{\partial U_{n 2}}{\partial t}+\frac{\partial U_{21}}{\partial x_{1}}+\ldots \frac{\partial U_{11}}{\partial x_{n-1}}=0
\end{array}\right. \\
& p^{j}:\left\{\begin{array}{l}
\frac{\partial U_{1 j}}{\partial t}+\frac{\partial U_{2 j-1}}{\partial x_{1}}+\ldots+\frac{\partial U_{n j-1}}{\partial x_{n-1}}=0 \\
\frac{\partial U_{2 j}}{\partial t}+\frac{\partial U_{1 j-1}}{\partial x_{1}}+\ldots+\frac{\partial U_{n j-1}}{\partial x_{n-1}}=0 \\
\vdots \\
\frac{\partial U_{n j}}{\partial t}+\frac{\partial U_{2 j-1}}{\partial x_{1}}+\ldots+\frac{\partial U_{1 j-1}}{\partial x_{n-1}}=0
\end{array}\right.
\end{aligned}
$$

For simplicity we have:

$$
\begin{aligned}
& U_{10}\left(x_{1}, x_{1}, \ldots, x_{n-1}, 0\right)=u_{10}\left(x_{1}, x_{1}, \ldots, x_{n-1}, t\right)=f_{1}\left(x_{1}, x_{1}, \ldots, x_{n-1}\right), \\
& U_{20}\left(x_{1}, x_{1}, \ldots, x_{n-1}, 0\right)=u_{20}\left(x_{1}, x_{1}, \ldots, x_{n-1}, t\right)=f_{2}\left(x_{1}, x_{1}, \ldots, x_{n-1}\right), \\
& \vdots \\
& U_{n 0}\left(x_{1}, x_{1}, \ldots, x_{n-1}, 0\right)=u_{n 0}\left(x_{1}, x_{1}, \ldots, x_{n-1}, t\right)=f_{n}\left(x_{1}, x_{1}, \ldots, x_{n-1}\right) .
\end{aligned}
$$

We have the following scheme:

$$
\begin{aligned}
& U_{11}(x, t)=-\int_{0}^{t}\left(\frac{\partial U_{20}}{\partial x_{1}}+\ldots+\frac{\partial U_{n 0}}{\partial x_{n-1}}-g_{1}\right) d t \\
& U_{21}(x, t)=-\int_{0}^{t}\left(\frac{\partial U_{10}}{\partial x_{1}}+\ldots+\frac{\partial U_{n 0}}{\partial x_{n-1}}-g_{2}\right) d t \\
& \vdots \\
& U_{n 1}(x, t)=-\int_{0}^{t}\left(\frac{\partial U_{20}}{\partial x_{1}}+\ldots+\frac{\partial U_{10}}{\partial x_{n-1}}-g_{n}\right) d t .
\end{aligned}
$$

Having this assumption we get the following iterative equations: 


$$
\begin{aligned}
& U_{1 j}(x, t)=-\int_{0}^{t}\left(\frac{\partial U_{2 j-1}}{\partial x_{1}}+\ldots+\frac{\partial U_{n 0}}{\partial x_{n-1}}-g_{1}\right) d t, \quad j=2,3, \ldots \\
& U_{2 j}(x, t)=-\int_{0}^{t}\left(\frac{\partial U_{1 j-1}}{\partial x_{1}}+\ldots+\frac{\partial U_{n j-1}}{\partial x_{n-1}}-g_{2}\right) d t, \quad j=2,3, \ldots \\
& \vdots \\
& U_{n j}(x, t)=-\int_{0}^{t}\left(\frac{\partial U_{2 j-1}}{\partial x_{1}}+\ldots+\frac{\partial U_{1 j-1}}{\partial x_{n-1}}-g_{n}\right) d t . \quad j=2,3, \ldots
\end{aligned}
$$

The approximate solution of (10) can be obtained by setting $p=1$ :

$$
\begin{aligned}
& u_{1}=\lim _{p \rightarrow 1} U_{1}=U_{10}+U_{11}+U_{12}+\ldots \\
& u_{2}=\lim _{p \rightarrow 1} U_{2}=U_{20}+U_{21}+U_{22}+\ldots \\
& \vdots \\
& u_{n}=\lim _{p \rightarrow 1} U_{n}=U_{n 0}+U_{n 1}+U_{n 2}+\ldots
\end{aligned}
$$

Now to solve the system (10), by Adomian decomposition method constructs the following forms of this method [6, 7]:

$$
\begin{aligned}
& U_{1}\left(x_{1}, x_{2}, \ldots, x_{n-1}, t\right)=u_{1}\left(x_{1}, \ldots, x_{n-1}, 0\right)+\int_{0}^{t} g_{1}\left(x_{1}, \ldots, x_{n-1}\right) d t+\int_{0}^{t}\left(\frac{\partial U_{2}}{\partial x_{1}}+\ldots+\frac{\partial U_{n}}{\partial x_{n-1}}\right) d t, \\
& U_{2}\left(x_{1}, x_{2}, \ldots, x_{n-1}, t\right)=u_{2}\left(x_{1}, \ldots, x_{n-1}, 0\right)+\int_{0}^{t} g_{2}\left(x_{1}, \ldots, x_{n-1}\right) d t+\int_{0}^{t}\left(\frac{\partial U_{1}}{\partial x_{1}}+\ldots+\frac{\partial U_{n}}{\partial x_{n-1}}\right) d t, \\
& \vdots \\
& U_{n}\left(x_{1}, x_{2}, \ldots, x_{n-1}, t\right)=u_{n}\left(x_{1}, \ldots, x_{n-1}, 0\right)+\int_{0}^{t} g_{n}\left(x_{1}, \ldots, x_{n-1}\right) d t+\int_{0}^{t}\left(\frac{\partial U_{2}}{\partial x_{1}}+\ldots+\frac{\partial U_{1}}{\partial x_{n-1}}\right) d t .
\end{aligned}
$$

To solve Eq. (13) by Adomian decomposition method let consider, as usual in this method, the solution $u$ as the summation of a series, say:

$u_{i n}=\sum_{n=0}^{\infty} u_{i n}, \quad i=1,2, \ldots$

And the integrand on the right sides as following series:

$$
\begin{aligned}
& \frac{\partial U_{2}}{\partial x_{1}}+\frac{\partial U_{3}}{\partial x_{2}}+\ldots+\frac{\partial U_{n}}{\partial x_{n-1}}=\sum_{n=0}^{\infty} A_{1 n}\left(u_{0}, u_{1}, . ., u_{n}\right), \\
& \frac{\partial U_{1}}{\partial x_{1}}+\frac{\partial U_{2}}{\partial x_{2}}+\ldots+\frac{\partial U_{n}}{\partial x_{n-1}}=\sum_{n=0}^{\infty} A_{2 n}\left(u_{0}, u_{1}, . ., u_{n}\right), \\
& \vdots \\
& \frac{\partial U_{2}}{\partial x_{1}}+\frac{\partial U_{3}}{\partial x_{2}}+\ldots+\frac{\partial U_{n}}{\partial x_{n-1}}=\sum_{n=0}^{\infty} A_{i n}\left(u_{0}, u_{1}, . ., u_{n}\right) . \quad i=1,2,3, . .
\end{aligned}
$$

Where $A_{i n}\left(u_{0}, u_{1}, \ldots, u_{n}\right), i=1,2, \ldots$ are called Adomian polynomials and should be computed. Substituting from (14) and (15) in to (13) we get:

$$
\begin{aligned}
& \sum_{i=1}^{\infty} U_{1 i}=f_{1}\left(x_{1}, \ldots, x_{n-1}\right)+\int_{0}^{t} g_{1}\left(x_{1}, \ldots, x_{n-1}\right) d t+\int_{0}^{t} \sum_{n=0}^{\infty} A_{1 n}\left(u_{0}, \ldots, u_{n}\right) d t \\
& \sum_{i=1}^{\infty} U_{2 i}=f_{2}\left(x_{1}, \ldots, x_{n-1}\right)+\int_{0}^{t} g_{2}\left(x_{1}, \ldots, x_{n-1}\right) d t+\int_{0}^{t} \sum_{n=0}^{\infty} A_{2 n}\left(u_{0}, \ldots, u_{n}\right) d t \\
& \vdots \\
& \sum_{i=1}^{\infty} U_{n i}=f_{n}\left(x_{1}, \ldots, x_{n-1}\right)+\int_{0}^{t} g_{n}\left(x_{1}, \ldots, x_{n-1}\right) d t+\int_{0}^{t} \sum_{n=0}^{\infty} A_{i n}\left(u_{0}, \ldots, u_{n}\right) d t . \quad i=1,2,3, \ldots
\end{aligned}
$$

From Eq. (16) the following procedure can be defined: 
$U_{0}=f_{0}\left(x_{1}, \ldots, x_{n-1}\right)+\int_{0}^{t} g_{0}\left(x_{1}, \ldots, x_{n-1}\right) d t$,

:

$U_{n+1}=-\int_{0}^{t} \sum_{n=0}^{\infty} A_{i n}\left(u_{0}, \ldots, u_{n}\right) d t . \quad i=1,2,3, \ldots$

So we can calculate the terms of, $u=\sum_{n=0}^{\infty} u_{n}$, term by term, as long as we derive the desired accuracy, the more terms the more accuracy.

Adomian polynomials have been calculated by using an alternate algorithm so,

$u_{1, n}=\int_{0}^{t}\left(\frac{\partial U_{2, n-1}}{\partial x_{1}}+\ldots+\frac{\partial U_{n, n-1}}{\partial x_{n}}\right) d t$,

$u_{2, n}=\int_{0}^{t}\left(\frac{\partial U_{1, n-1}}{\partial x_{1}}+\ldots+\frac{\partial U_{n, n-1}}{\partial x_{n}}\right) d t$

$\vdots$

$u_{n, n}=\int_{0}^{t}\left(\frac{\partial U_{2, n-1}}{\partial x_{1}}+\ldots+\frac{\partial U_{1, n-1}}{\partial x_{n}}\right) d t$

\section{Numerical Example}

Two examples are provided; these are considered to illustrate the Homotopy methods to solve a system of partial differential equations.

Example 1.Consider the following system of partial differential equation

$\frac{\partial u}{\partial t}-v\left(\frac{\partial u}{\partial x}\right)^{2}=0$

$\frac{\partial v}{\partial t}-u\left(\frac{\partial v}{\partial x}\right)^{2}=0$

With initial condition:

$u(x, 0)=e^{x}, v(x, 0)=e^{x}$

We construct the following homotopy $\Omega \times[0,1] \rightarrow \Re$, which satisfies:

$\frac{\partial U}{\partial t}-\frac{\partial u_{0}}{\partial t}+p\left(\frac{\partial u_{0}}{\partial t}-V\left(\frac{\partial U}{\partial x}\right)^{2}\right)=0$
$\frac{\partial V}{\partial t}-\frac{\partial v_{0}}{\partial t}+p\left(\frac{\partial v_{0}}{\partial t}-U\left(\frac{\partial V}{\partial x}\right)^{2}\right)=0$.

Let's consider the following initial approximation to the solution of Eq. (19),

$U_{0}(x, t)=u_{0}(x, t)=e^{x}$,

$V_{0}(x, t)=v_{0}(x, t)=e^{-x}$.

By these assumptions, one gives the following results,

$u_{1}=t e^{x}, v_{1}=t e^{-x}$,

$u_{2}=3 / 2 t^{2} e^{x}, v_{2}=3 / 2 t^{2} e^{-x}$,

$u_{3}=5 / 2 t^{3} e^{x}, v_{3}=5 / 2 t^{3} e^{-x}$,

$\vdots$

The series are obtained: 
$u(x, t)=\sum_{n=0}^{\infty} u_{n}(x, t)=\sum_{n=0}^{\infty} \alpha_{n} t^{n} e^{x}$,
$v(x, t)=\sum_{n=0}^{\infty} v_{n}(x, t)=\sum_{n=0}^{\infty} \alpha_{n} t^{n} e^{-x}$.

This is an exact solution. This solution is the same as the result obtained by Adomian Decomposition Method.

\section{Example2.}

Consider the fallowing system of inhomogeneous partial differential equation:

$\frac{\partial u}{\partial t}-\left(\frac{\partial v}{\partial x}\right)\left(\frac{\partial w}{\partial x}\right)=3 / 2-1 / 2\left(e^{-2 x}\right)$

$\frac{\partial v}{\partial t}-\left(\frac{\partial u}{\partial x}\right)\left(\frac{\partial w}{\partial x}\right)=3 / 2-1 / 2\left(e^{2 x}\right)$

$\frac{\partial w}{\partial t}-\left(\frac{\partial u}{\partial x}\right)\left(\frac{\partial v}{\partial x}\right)=2$

With the exact solutions:

$u(x, t)=e^{x}+t$

$v(x, t)=e^{-x}+t$,

$w(x, t)=1 / 2\left(e^{x}+e^{-x}\right)+t$.

And with initial condition:

$u(x, 0)=e^{x}$,

$v(x, 0)=e^{-x}$,

$w(x, 0)=1 / 2\left(e^{x}+e^{-x}\right)$.

The Adomian decomposition can be readily constructed as

$u(x, t)=\int_{0}^{t}\left(e^{x}+3 / 2-1 / 2\left(e^{-2 x}\right)\right) d t+\int_{0}^{t} \sum_{k=0}^{n}\left(A_{k}\left(u_{0}, . ., u_{k}\right)\right) d t$,

$v(x, t)=\int_{0}^{t}\left(e^{-x}+3 / 2-1 / 2\left(e^{2 x}\right)\right) d t+\int_{0}^{t} \sum_{k=0}^{n}\left(A_{k}\left(v_{0}, . ., v_{k}\right)\right) d t$,

$w(x, t)=\int_{0}^{t}\left(2+1 / 2\left(e^{x}+e^{-x}\right)\right) d t+\int_{0}^{t} \sum_{k=0}^{n}\left(A_{k}\left(w_{0}, . ., w_{k}\right)\right) d t$.

Let's take initial condition, as initial approximations

$U_{0}(x, t)=u_{0}(x, t)=e^{x}$,

$V_{0}(x, t)=v_{0}(x, t)=e^{-x}$,

$W_{0}(x, t)=w_{0}(x, t)=1 / 2\left(e^{x}+e^{-x}\right)$.

Therefore we have

$A\left(u_{0}\right)=-1 / 2+1 / 2\left(e^{-2 x}\right)-1 / 2\left(e^{3 x}\right)+1 / 2 t\left(e^{x}\right)$,

$A\left(v_{0}\right)=-1 / 2+1 / 2\left(e^{2 x}\right)-1 / 2\left(e^{-3 x}\right)+1 / 2 t\left(e^{-x}\right)$,

$A\left(w_{0}\right)=-1-t\left(e^{-3 x}\right)-t\left(e^{3 x}\right)-t^{2}$.

$\vdots$

$u_{1}=-1 / 4 t^{2} e^{3 x}+1 / 4 t^{2} e^{x}-1 / 2 t+1 / 2 t e^{-2 x}$,

$v_{1}=-1 / 4 t^{2} e^{-3 x}+1 / 4 t^{2} e^{-x}+1 / 2 t e^{x}-1 / 2 t$,

$w_{1}=-t-1 / 3 t^{3}-1 / 2 t^{2} e^{-3 x}-1 / 2 t^{2} e^{3 x}$.

:

And the series solution will be derived by adding these terms as: 


$$
\begin{aligned}
& u(x, t)=\sum_{n=0}^{\infty} u_{n}(x, t), \\
& v(x, t)=\sum_{n=0}^{\infty} v_{n}(x, t), \\
& w(x, t)=\sum_{n=0}^{\infty} w_{n}(x, t) .
\end{aligned}
$$

Some value of the eight terms approximation to the solutions are presented in the following Tables 1, 2, 3 .

Table 1: the ADM results for $\mathrm{u}(\mathrm{x}, \mathrm{t})$

\begin{tabular}{cccc}
\hline$x$ & $t$ & $u_{A D M}$ & $e(u(n=8))$ \\
\hline-0.0052 & -0.0888 & 0.9060135351 & $0.394 \mathrm{e}-6$ \\
-0.0325 & -0.0888 & 0.8792226799 & $0.230 \mathrm{e}-5$ \\
-0.0888 & -0.0888 & 0.8262291480 & $0.597 \mathrm{e}-5$ \\
-0.0980 & -0.0888 & 0.8178495454 & $0.441 \mathrm{e}-5$ \\
\hline
\end{tabular}

Table 2: the ADM results for $\mathrm{v}(\mathrm{x}, \mathrm{t})$

\begin{tabular}{cccc}
\hline$x$ & $t$ & $v_{A D M}$ & $e(v(n=8))$ \\
\hline-0.0052 & -0.0888 & 0.91641350380 & $0.392 \mathrm{e}-6$ \\
-0.0325 & -0.0888 & 0.94422336351 & $0.275 \mathrm{e}-5$ \\
-0.0888 & -0.0888 & 0.00406125900 & $0.803 \mathrm{e}-6$ \\
-0.0980 & -0.0888 & 0.01416187700 & $0.908 \mathrm{e}-6$ \\
\hline
\end{tabular}

Table 3: the ADM results for $\mathrm{w}(\mathrm{x}, \mathrm{t})$

\begin{tabular}{cccc}
\hline$x$ & $t$ & $w_{A D M}$ & $e(w(n=8))$ \\
\hline-0.0052 & -0.0888 & 0.91121153210 & $0.199 \mathrm{e}-4$ \\
-0.0325 & -0.0888 & 0.91172613388 & $0.203 \mathrm{e}-4$ \\
-0.0888 & -0.0888 & 0.91514296970 & $0.342 \mathrm{e}-5$ \\
-0.0980 & -0.0888 & 0.91600342190 & $0.242 \mathrm{e}-4$ \\
\hline
\end{tabular}

\section{Conclusion}

In this paper, we have applied Homotopy analysis method and Adomian decomposition method for solving systems of partial differential equations. Examples show that the analytical approximation to the solutions is reliable.Ability of these 2 methods as an easy device for computing the solution of the systems of PDE [8].

\section{References}

[1] J. H He, Homotopy perturbation technique, computer Methods in Applied Mechanics and Engineering 178 (1999) $257-262$.

[2] J. H He, A coupling method of Homotopy technique and perturbation technique for nonlinear problems, International Journal of Non-Linear Mechanics 35 (1) (2003) 7-43.

[3] G. Adomian, Nonlinear Stochastic Systems Theory and Applications to Physics', Khuwer Academic Press, (1989).

[4] G. Adomian, solving Frontier problem of physics: the Decomposition Method, Khuwer Academic Press, (1994).

[5] Ch. K. Chen and Sh. H Ho, solving partial differential equation by two-dimensional Differentional transform method, Applied Mathematics and computation 106 (1999) 171-179.

[6] G. Adomian, Review of the decomposition method in applied Mathematics, J. Math. Anal, Apply, 135 (1988) .501-544.

[7] Y. Cherruault, Convergence of Adomian method, Kybernets, 18 (1989) 31-38.

[8] Abdul- Majid Wazwaz, the existence of noise terms for system of inhomogeneous differential and integral equations Applied Mathematics and Computation 146 (2003) 81-92. 